\title{
What if the Ventricle was Innocent for Once? The Infrequent Case of a Guilty Atrium
}

\author{
Angelica Abbate $^{1 *}$, Giulia Teresi ${ }^{1}$, Davide Piraino ${ }^{1}$, Giuseppe Andolina ${ }^{1}$ and Pasquale Assennato $^{2}$ \\ ${ }^{1}$ Section of Interventional Cardiology and Hemodynamic, A.O.U.P "Paolo Giaccone", Palermo, Italy \\ ${ }^{2}$ Division of Cardiology, Cardiovascular Diseases, Department of Internal Medicine, Cardiovascular and \\ Nephrologic Diseases, Paolo Giaccone Hospital, University of Palermo, Italy
}

*Corresponding author: Angelica Abbate, Section of Interventional Cardiology and Hemodynamic, A.O.U.P "Paolo Giaccone", Palermo, Italy, Tel: +393209480649, E-mail: angelicabbate@libero.it

\begin{abstract}
Abbreviations
ECG: Electrocardiogram; EF: Ejection Fraction; GLA: Giant Left Atrium; LA: Left Atrial/Atrium; LV: Left Ventricle/Ventricular; RA: Right Atrium; RV: Right Ventricle; TTE: Transthoracic Echocardiography
\end{abstract}

\section{Dear Editor,}

Giant left atrium (GLA) is a rare disease (reported incidence of $0.3 \%)$, generally occurs in the mitral valve disease, chronic atrial fibrillation, in patent ductus arteriosus and ventricular septal defect [1,2] and correlated symptoms as dyspnea and dysphagia are a specific [3].

The transthoracic echocardiography (TTE) is the most sensitive imaging technique to diagnose such a rare case, requiring an atrial anteroposterior diameter greater than $80 \mathrm{~mm}$ [4] measured in the parasternal long axis view.

An 82-year-old man with chronic atrial fibrillation was admitted to our department for progressive dyspnea, III NYHA class. Almost 10 years ago, he underwent replacing of mitral valve with a mechanical prosthesis CarboMedics size 31 (Sorin Group - Milan, Italy) due to severe mitral valve insufficiency and plastic of tricuspid valve due to moderate - severe insufficiency.

The post-operative TTE showed normal size and wall motion of the left ventricle (LV), mechanical mitral valve prosthesis without paraprosthetic regurgitation, ejection fraction (EF) of $55 \%$ and dilated left atrium (LA) with an anteroposterior diameter of $61 \mathrm{~mm}$. The patient has had a follow-up every six month for the first year and then every year, in which echocardiographic assessments were stable.

At admission, he had normal hemodynamic parameters. The electrocardiogram (ECG) showed bradyarrhythmia for chronic atrial fibrillation with heart rate of $55 \mathrm{bpm}$.

Although the disabling dyspnea, TTE showed a normal LV segmental kinesis, but a surprising abnormal LA dilatation with a significant $L V$ compression by the huge LA (Figure $1 \mathrm{~A}$ and Figure 1B). The estimated LA volume was between 822.6 and $1035.1 \mathrm{ml}$ with an anteroposterior diameter between 141.08 and $149.59 \mathrm{~mm}$ (Figure $1 \mathrm{C}$ and Figure 1D). Impossible to estimate correctly EF. Absence of pericardial effusion. Systolic pulmonary artery pressure was $40 \mathrm{mmHg}$. There was also right atrium (RA) dilatation (Figure 2).

Literature suggests that LA dilatation occurs in response to pressure overload resulting from fibrosis and/ or calcification of the LA, increased LA afterload, in the setting of mitral valve disease or LV dysfunction [5], but in our case LV dysfunction is a consequence of crushing by huge LA. The literature does not contain case report in this regard about the GLA that compresses the LV, nor a LA anteroposterior diameter $>130 \mathrm{~mm}$ [6].

Left atrial plication, among other surgical techniques to reduce LA dimensions, seems the most suitable, although some surgeons established that the mitral valve 


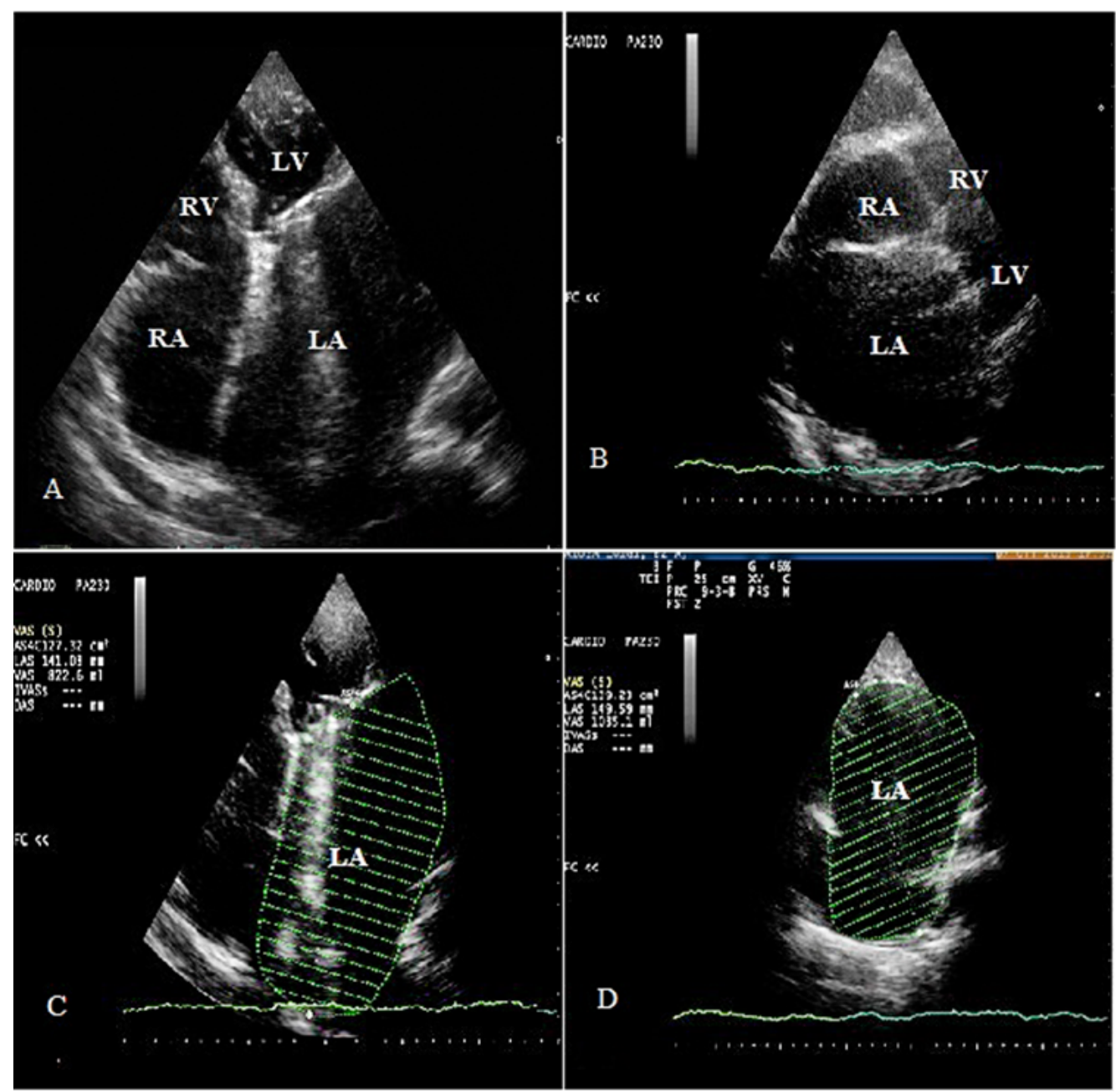

Figure 1: A) Apical four-chamber view of the LV compressed by the huge LA and mechanical mitral valve prosthesis; $B$ ) Subcostal four-chamber view of the enormous LA; absence of pericardial effusion; C,D) Apical four-chamber view of the LA dimensions.

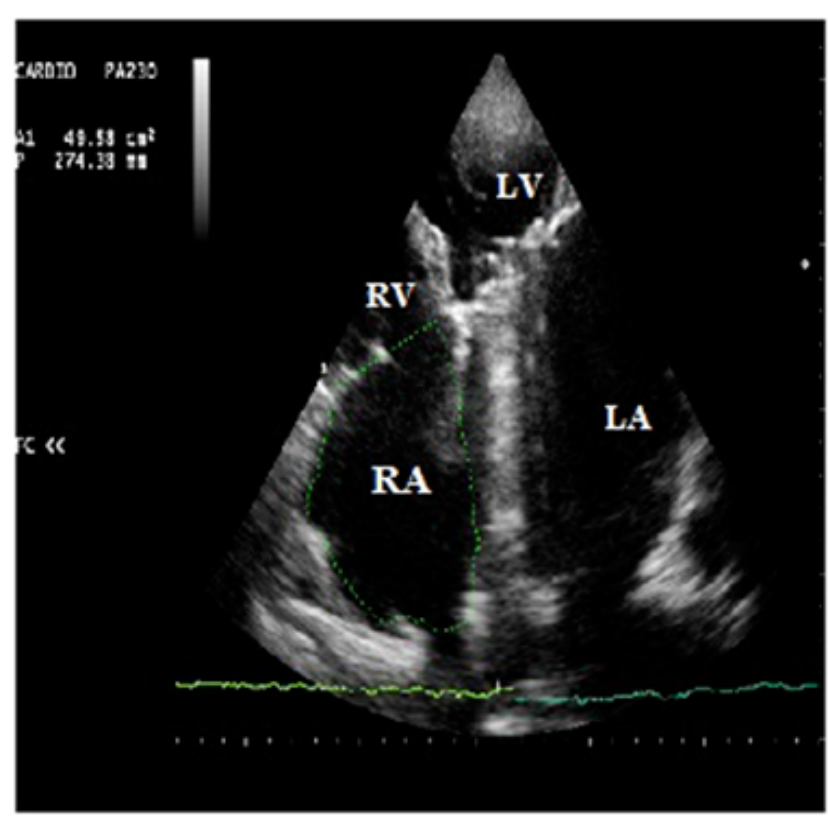

Figure 2: Apical four-chamber view: Highlights increased size of RA.

surgery alone will result in the reduction of left atrial size. Nevertheless, the rheumatic and irreversible process causes loss of tone by the elastic fibers of the tis- sue; Thus, the left atrial enlargement is beyond repair [7]. Atrial fibrillation also may be a possible cause of atrial enlargement since experimental animal studies have documented that it induces electrical, contractile and structural remodeling [8].

The normal wall motion of the LV suggests that our patient is affected by an atypical dyspnea because it is not induced by LV diastolic dysfunction [9]. First reported in literature, the enormous atrium of our patient (anteroposterior diameter between 141.08 and $149.59 \mathrm{~mm}$ ) plays an important role in determining the ingravescent dyspnea: The GLA crushes the LV that involves incomplete and inadequate ventricular filling. The LV compression probably induces not only diastolic dysfunction but also systolic one, since it was difficult to estimate the effective FE just because the LV is crushed.

\section{References}

1. L Di Marco, L Careddu, E Pilato, R Di Bartolomeo (2012) Atrio sinistro gigante con stenosi mitralica reumatica. $G$ Ital Cardiol 13: 523-524.

2. Fasseas $P$, Lee-Dorn $R$, Sokil $A B$, Van Decker W (2001) Giant left atrium. Tex Heart Inst J 28: 158-159.

3. Hurst JW (2001) Memories of patients with a giant left atrium. Circulation 104: 2630-2631. 
4. Okyay K, Cengel A, Tavil Y (2007) A giant left atrium with two huge thrombi without embolic complications. Can $\mathrm{J}$ Cardiol 23: 1088.

5. Walter P, Seward JB, Appleton CP, Douglas PS, Oh JK, et al. (2006) Left atrial size: Physiologic determinants and clinical applications. J Am Coll Cardiol 20: 2357-2363.

6. Okan Gulel, Diyar Koprulu, Zafer Kucuksu, Mustafa Yazici, Senem Cengel (2007) Cardiovocal syndrome associated with huge left atrium. Circulation 115: e318-e319.
7. Apostolakis E, Shuhaiber JH (2008) The surgical management of giant left atrium. Eur J Cardiothorac Surg 33: 182-190.

8. Allessie M, Ausma J, Schotten U (2002) Electrical, contractile and structural remodeling during atrial fibrillation. Cardiovasc Res 54: 230-246.

9. Teo SG, Yang H, Chai P, Yeo TC (2010) Impact of left ventricular diastolic dysfunction on left atrial volume and function: A volumetric analysis. Eur J Echocard 11: 38-43. 